\title{
Derivatives obtained directly from displacement data
}

\author{
Y. Zou, G. Pedrini, H. Tiziani \\ Institute of Applied Optics, University of Stuttgart, Pfaffenwaldring 9, 70569 Stuttgart, Germany
}

Received 4 April 1994; revised manuscript received 10 August 1994

\begin{abstract}
Two flexible shearing methods taking the derivative information directly from the data for displacement measurement are reported. The displacement information is obtained using a holographic recording directly on a CCD (charge-coupled-device) chip. Besides the advantage of taking the displacement information and the derivative information from a same set of data, other characteristics of these methods are that the derivative sensitivity can be controlled after recordings and displacement derivatives along arbitrary directions can be chosen easily. One method is based on the electronic recording which keeps the complex amplitudes of the wave fronts in a computer. The other needs only the phase modulo $2 \pi$ of the displacement.
\end{abstract}

\section{Introduction}

For strain analyses, the derivatives of displacements are needed. The derivatives can be obtained by either numerical differentiation of the displacement data or some shearographic techniques [1,2]. For a derivative in a certain direction, an optical arrangement with the appropriate shear direction is required [3] or a defocusing method is employed [4]. Some shearing technique can give the information of the derivatives of displacement and the displacement by switching the measurement from one to another [5]. To obtain the displacement information and its derivatives simultaneously a multi-aperture set-up [6] or two recording systems [5] were used. Apart from the numerical differentiation method, second derivatives of the deflection which present a major interest related to the bending moment and to the stresses can be obtained by setting the second device in a shearing system [7]. The phase evaluation in shearing techniques is normally made by using phase-shifting methods [8] which introduce additional errors and/or additional experimental efforts.
In the presented paper two simple digital shearing methods with great flexibility are reported. One is based on a recently presented electronic holography procedure [9]. By introducing artificially a linear shift on the image plane in the reconstruction, a shearogram is obtained directly from displacement measurement data. The phase of each state for producing a shearogram can be calculated directly from the complex amplitudes stored in the computer. The other is a more general method where the phase derivatives are obtained directly from displacement phase data obtained from a displacement sensitive measurement procedue. It is actually a data pocessing in a frame grabber with a computer. The phase of the shearogram is performed by making a shear of the modulo $2 \pi$ phase map of the displacement. A higher derivative can be obtained by taking a second shear of the sheared modulo $2 \pi$ phase map. In principle the information of all derivatives can be obtained from two post recordings of the wave fronts before and after displacement. Examples of lateral shear, radial shear, rotational shear, inversion shear and reversal shear [3] of a displacement are given, using the second method. 


\section{Principle of the shearing methods}

\subsection{Recording a hologram electronically}

With the rapidly developed CCD camera techniques, optical detectors with a great number of small sensor pixels are commercially available. It is now possible to record a hologram directly on a $\mathrm{CCD}$ chip. The interference between the object beam and the reference beam is registered electronically as an intensity modulation on the detector. The signal is then taken by a frame grabber and transferred to data array accessible by a computer. If the object wave is $\mathbf{A}_{0}(\boldsymbol{r})$ and the reference wave is $\mathbf{A}_{\mathbf{r}}(\boldsymbol{r})$ on the CCD plane, the intensity modulation can be represented as:

$$
\begin{aligned}
I & =\left|\mathbf{A}_{\mathrm{o}}(\boldsymbol{r})+\mathbf{A}_{\mathrm{r}}(\boldsymbol{r})\right|^{2} \\
& =\left|\mathbf{A}_{\mathrm{o}}\right|^{2}+\left|\mathbf{A}_{\mathrm{r}}\right|^{2}+\mathbf{A}_{\mathbf{r}} \mathbf{A}_{\mathrm{o}}{ }^{*}+\mathbf{A}_{\mathrm{o}} \mathbf{A}_{\mathbf{r}}{ }^{*},
\end{aligned}
$$

where $*$ denotes the complex conjugate amplitudes. The CCD array should resolve the interference with the maximum angle $\theta$ between the reference and the object beam. By simulating the diffraction of the hologram illuminated by the same reference wave in the computer, the object wave front can be reconstructed electronically. This technique does not need film or plate development and the replacement of the hologram as in conventional holography. The amplitude and the phase information of the reconstructed wave front are digitally stored in its real as well as in its imaginary part in the computer. This technique has another particular advantage when it is used for interferometry, namely the phase of a displacement can be directly obtained from the complex amplitude without applying any phase-shifting algorithms [9].

\subsection{Shearograms from post recordings}

For interferometry two recordings of two states of the object are needed. The complex amplitudes from both states are stored in a computer. In the case of out-of-plane displacement the states before and after the displacement are recorded. Because they can be separated by means of a computer storage, numerical manipulations between them are possible. After the numerical reconstruction of the wave front a lateral shift on it can be introduced. By adding or subtracting the shifted wave front onto the unshifted wave front, a

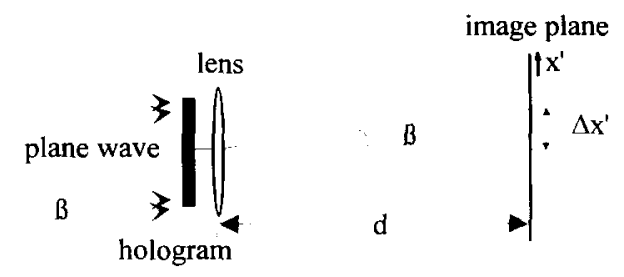

Fig. 1. Relation between a tilt of the reference wave and the shift of the image.

shear in this shift direction is obtained. From the numerical standpoint, a simple way to get a shear is to change the direction of the reference wave by computation during the reconstruction. A shift in the image plane corresponding to a tilt of the reference wave is (see Fig. 1)

$\Delta x^{\prime}=d \tan (\beta)$,

where $\Delta x^{\prime}$ is a shift in $x^{\prime}$-direction in the image plane, $\beta$ is the tilt angle of the reference wave front rotated along the $y$-axis and $d$ is the distance between the lens and the image plane. A shear in $x$-direction can be introduced by reconstructing the combination of the untilted and the tilted hologram. Comparing the intensities of the sheared image before displacement and the sheared image after displacement, a shearogram which represents a directional derivative is obtained. If $\phi_{0}(r)$ is the phase of the undeformed- and $\phi_{d}(r)$ the phase of the deformed object, the complex amplitudes are

$$
\begin{aligned}
& \mathbf{A}_{j}(\boldsymbol{r})=A_{j}(\boldsymbol{r}) \exp \left[\mathrm{i} \phi_{j}(\boldsymbol{r})\right], \\
& \mathbf{A}_{j}(\boldsymbol{r}+\Delta \boldsymbol{r})=A_{j}(\boldsymbol{r}+\Delta \boldsymbol{r}) \exp \left[\mathrm{i} \phi_{j}(\boldsymbol{r}+\Delta \boldsymbol{r})\right],
\end{aligned}
$$

where the subscript $j=0$ or $\mathrm{d}$ which indicates the state before or after the deformation, respectively, $r$ is a coordinate vector for a certain point on the image and $\Delta r$ is a shift of the image in a certain direction; $A_{j}(r)=$ $\left|\mathbf{A}_{j}(\boldsymbol{r})\right|$ and $A_{j}(\boldsymbol{r}+\Delta \boldsymbol{r})=\left|\mathbf{A}_{j}(\boldsymbol{r}+\Delta \boldsymbol{r})\right|$ are the moduli of the amplitudes. For a small shear, i.e. $A_{j}(r)=$ $A_{j}(\boldsymbol{r}+\Delta \boldsymbol{r})$, which provides only a phase change but not an amplitude change between two sheared points, the intensities of the sheared images before and after deformation can approximately be written as

$$
\begin{gathered}
I_{j}(\boldsymbol{r})=A_{j}^{2}\left|\exp \left[\mathrm{i} \phi_{j}(\boldsymbol{r})\right]+\exp \left[i \phi_{j}(\boldsymbol{r}+\Delta \boldsymbol{r})\right]\right|^{2} \\
=2 A_{j}^{2}\left\{1+\cos \left[\phi_{j}(\boldsymbol{r})-\phi_{j}(\boldsymbol{r}+\Delta \boldsymbol{r})\right]\right\},
\end{gathered}
$$


where $\Delta \boldsymbol{r}$ is the shear in an arbitrary direction. A small displacement provides fast phase changes but slight amplitude changes. Hence we have $A_{0}(r) \approx A_{\mathrm{d}}(r)=$ $A(r)$. Subtracing these two intensity patterns leads to

$$
\begin{aligned}
& I_{0}(\boldsymbol{r})-I_{\mathrm{d}}(\boldsymbol{r})=4 A^{2} \\
& \quad \times \sin \left(\frac{\phi_{\mathrm{o}}(\boldsymbol{r})+\phi_{\mathrm{d}}(\boldsymbol{r})-\phi_{\mathrm{o}}(\boldsymbol{r}+\Delta \boldsymbol{r})-\phi_{\mathrm{d}}(\boldsymbol{r}+\Delta \boldsymbol{r})}{2}\right) \\
& \quad \times \sin \left(\frac{\Delta \Phi(\boldsymbol{r})}{2}\right),
\end{aligned}
$$

where $\Delta \Phi(\boldsymbol{r})=\left[\phi_{\mathrm{o}}(\boldsymbol{r})-\phi_{\mathrm{o}}(\boldsymbol{r}+\Delta \boldsymbol{r})\right]-\left[\boldsymbol{\phi}_{\mathrm{d}}(\boldsymbol{r})-\right.$ $\left.\phi_{\mathrm{d}}(\boldsymbol{r}+\Delta \boldsymbol{r})\right]$, represents the derivative of the displacement phase. The absolute value of Eq. (5) is normally stored in a frame grabber and can be displayed on a monitor. The first sin term is a fast modulation and is immersed in speckles. The second term produces the shearing fringes. The direction and the sensitivity of a derivative can easily be controlled by altering the tilting angle of the reference wave in the reconstruction.

\subsection{Derivatives from complex amplitudes}

The digital reconstruction of the complex amplitude contains the real as well as the imaginary part in arrays. The phases of four object states related to two original recordings and two modifications of them (a linear phase shift on them) can be calculated directly from their complex amplitudes. For instance,

$\phi_{j}(\boldsymbol{r})=\arctan \frac{\operatorname{Im}\left[\mathbf{A}_{j}(\boldsymbol{r})\right]}{\operatorname{Re}\left[\mathbf{A}_{j}(\boldsymbol{r})\right]}$,
$\phi_{j}(\boldsymbol{r}+\Delta \boldsymbol{r})=\arctan \frac{\operatorname{Im}\left[\mathbf{A}_{j}(\boldsymbol{r}+\Delta \boldsymbol{r})\right]}{\left.\operatorname{Re}\left[\mathbf{A}_{j}(\boldsymbol{r})+\Delta \boldsymbol{r}\right)\right]}$,

where $j=o$ or d, represents without or with deformation, respectively; Re denotes the real part and Im the imaginary part. By taking the signs of the real and the imaginary parts into account the phases have modulo $2 \pi$. A difference between them represents the derivative of the displacement [2]:

$$
\begin{aligned}
\Delta \Phi & =\left[\phi_{\mathrm{o}}(\boldsymbol{r})-\phi_{\mathrm{d}}(\boldsymbol{r})\right]-\left[\phi_{\mathrm{o}}(\boldsymbol{r}+\Delta \boldsymbol{r})-\phi_{\mathrm{d}}(\boldsymbol{r}+\Delta \boldsymbol{r})\right] \\
& =\frac{2 \pi}{\lambda} \frac{\partial w}{\partial r} \Delta r
\end{aligned}
$$

where $w$ is an out-of-plane displacement, $\partial w / \partial r$ is a directional derivative of the displacement in $r$-direction.
Because the phases are calculated separately from the complex amplitudes but not the intensities, it is unnecessary to assume $A_{j}(r)=A_{j}(r+\Delta r)$ to get Eq. (4) and $A_{\mathrm{o}}(r) \approx A_{\mathrm{d}}(r)$ to obtain Eq. (5). A shearogram which depends on the intensities of the recordings plays no role in the phase calculation, but it can probably be used for controlling the direction and the amount of the shearing before the phase calculation, because the calculations of four wave fronts take a relative long time.

\subsection{Another alternative to differentiate the displacement data}

When the phase modulo $2 \pi$ of a displacement is known from any measurement, a direct shear of this pattern can give the derivative information. If $\phi_{0}(r)-$ $\phi_{\mathrm{d}}(r)$ presents the phase modulo $2 \pi$ of the displacement, making a shift of this phase map in the frame grabber and then subtracting it from the unshifted phase map, one obtains Eq. (7). The direction and the sensitivity of the derivative are controlled by altering the shearing direction and the amount. This procedure is also valid for a higher order derivative, for instance for $\partial^{2} w / \partial x^{2}, \partial^{2} w / \partial x \partial y, \partial^{2} w / \partial y^{2}$, etc. It is in principle a differentiation method. But differing from the conventional numerical differentiation method, it makes a discrete derivation of a sampled image before the phase unwrapping. It needs only simple image processing in the frame grabber like shift, rotation, contraction, subtraction, etc.. The resulting derivatives with phase modulo $2 \pi$ directly show the directions and the sensitivities of the shearing.

When the phase map with modulo $2 \pi$ represents the topographic shape information of a three-dimensional object the information on the surface slope variations

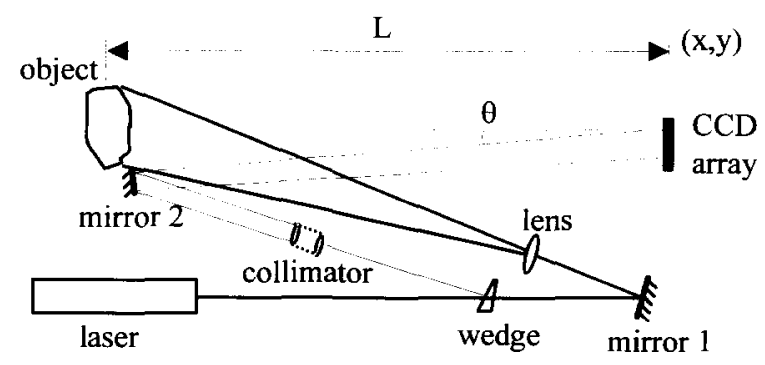

Fig. 2. Experimental set-up for holographic recording on a CCD array. 


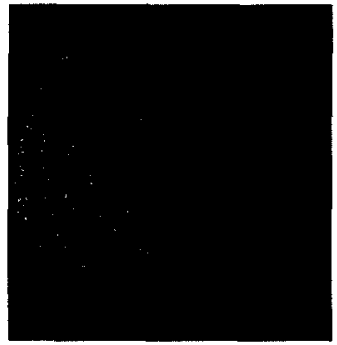

a)

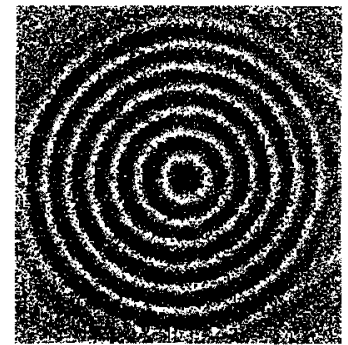

b)

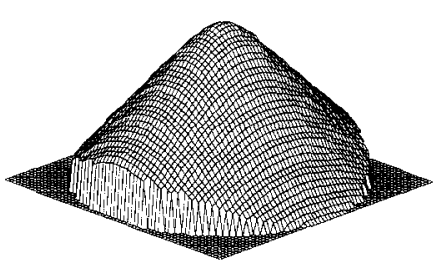

c)

Fig. 3. Displacement measurement of a centrally loaded object: (a) electronically reconstructed interferogram; (b) phase modulo $2 \pi$ of the interferogram and (c) pseudo $3 \mathrm{D}$ plot of the deformation.

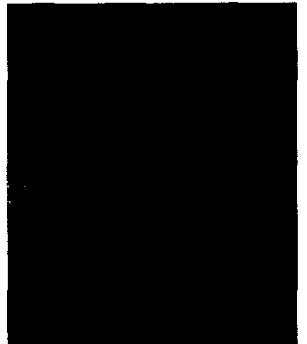

a)

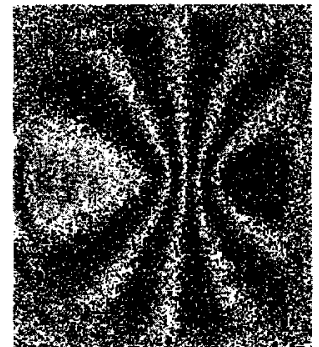

b)

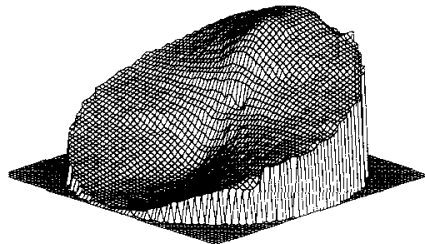

c)

Fig. 4. Derivative measurement from an electronically recorded hologram: (a) shearogram; (b) phase modulo $2 \pi$ of the shearogram and (c) the $3 \mathrm{D}$ plot of the derivative.

of this object can be obtained by shearing the phase map.

\section{Experimental results}

A simple set-up for the electronic holographic recording is schematically shown in Fig. 2. The detector was a normal interline transfer CCD camera with $768 \times 512$ sensor pixels. The light source was a diode pumped, frequency doubled $\mathrm{Nd}^{+}$YAG laser of which only $15 \mathrm{~mW}$ power was used for the exposures. The beam from laser was split into a reference wave and an object illumination wave by a glass wedge. The mirror 2 was close to the object and the object was placed far away from the detector in order to get resolvable interference fringes on the CCD array. The distance between the object and the detector was $176 \mathrm{~cm}$ and the maximum angle between the reference and the object ray was about $28 \mathrm{mrad}$. For $\lambda=0.533 \mu \mathrm{m}$ the maximum spatial frequency of the interference was about $(18.8 \mu \mathrm{m})^{-1}$, which was almost resolved by our camera (pixel size of $11 \mu \mathrm{m}$ ). The object was an edge-clamped and centre-loaded circular diaphragm. The illumination area was about $3.5 \mathrm{~cm}$ in diameter. Two holograms were rernrded hefore and after the loading, respectively. Reconstruction of a hologram was performed with a standard FFT program and the virtual image was used for processing. Subtracting the two wave fronts reconstructed from both holograms, an interferogram was obtained as seen in Fig. 3a. To get the phase of the displacement the phases of two recordings were calculated from $\mathrm{Eq}$. (6) and were subtracted from each other. The phase modulo $2 \pi$ of the displacement and its pseudo 3D plot are shown in Fig. 3b and Fig. 3c, respectively. A tilt angle of $0.5 \mathrm{rad}$ of the reference wave about the $y$-axis (corresponding to a shift of $5 \mathrm{~mm}$ on the image plane in $x$-direction) was introduced. Operations along Eq. (4) to Eq. (5) were made. A shearogram obtained is shown in Fig. 4a. The calculated phase from Eqs. (6) - (7) and the pseudo 3D plot of the derivative are depicted in Fig. $4 \mathrm{~b}$ and Fig. 4c, respectively. The relative poor contrast of the shearogram was due to the insufficient equal amplitude assumptions. But the phase map has still high quality because it did not need those as- 


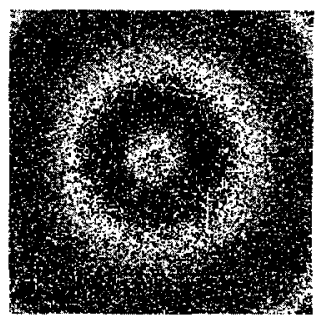

a)

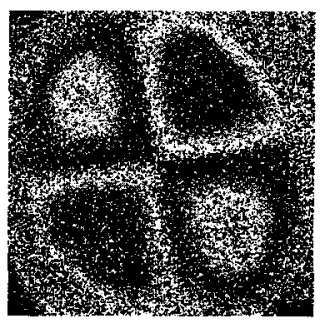

b)

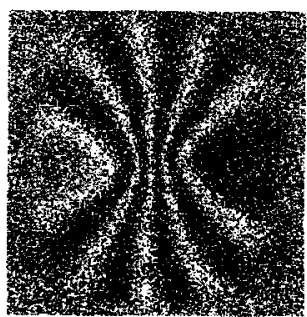

c)

Fig. 5. Phase maps of different shears: (a) radial shearing; (b) rotational shearing and (c) inversion shearing on an eccentrically loaded object.

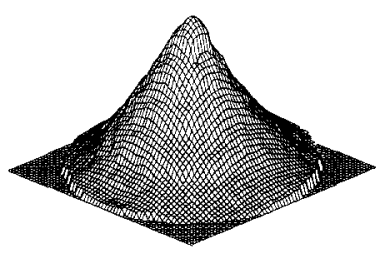

a)

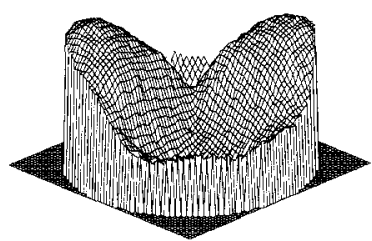

b)

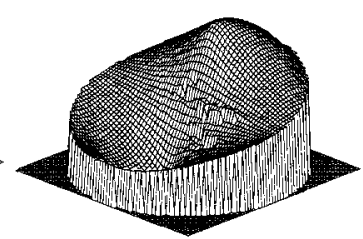

c)

Fig. 6. Pseudo 3D plots of: (a) the radial derivative; (b) the rotational derivative and (c) the inversion shear.

sumptions. There were no additional experimental components and no additional exposures, hence there were no additional externally introduced errors.

To show the second method a displacement phase map with modulo $2 \pi$ is needed from any displacement measurement procedure whatever. As an example, the phase map displayed in Fig. 3b was used. By making a shift operation of this pattern in a frame grabber (IMAGING TECHNOLOGY Inc.) and subtracting it from the original, the same pattern as in Fig. 4b was shown. For a radial shear the processing of the interferogram can be an image constracting or enlarging about its centre and then compared to its original. The result of a radial shear with $64 \%$ contracted image is shown in Fig. 5a. A rotational shear was introduced by rotating the interferogram around its centre and an inversion shear on an eccentrically loaded object was made by applying a $180^{\circ}$ rotational shear. The result of a rotational shear with $20^{\circ}$ clockwise rotated image and the result of a $5 \mathrm{~mm}$ eccentric inversion shear are presented in Fig. 5b and Fig. 5c, respectively. A reversal shear could be taken by folding the interferogram about a $y$-axis. Because the object was centrosymmetric and centrally loaded, no fringes are formed in the normal inversion shear and the normal reversal shear. With an eccentric loading the phase of the reversal

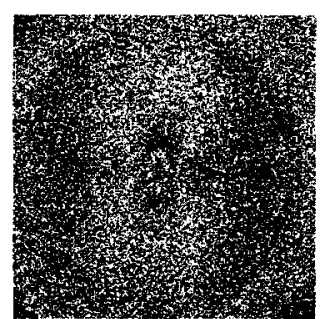

a)

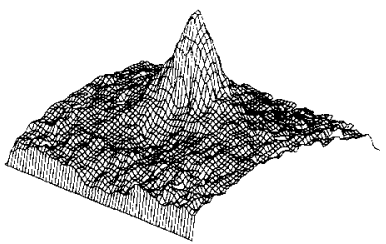

b)
Fig. 7. The second derivative in $x$-direction: (a) phase modulo $2 \pi$ and (b) the 3D plot.

shear was similar to the phase map shown in Fig. 5c. The pseudo 3D plots of the unwrapped phase maps from Figs. 5a-c are given in Figs. 6, accordingly. The second derivative in $x$-direction with phase modulo $2 \pi$ is indicated in Fig. 7a and was directly obtained from a shear of Fig. 4b. The pseudo 3D plot of the phase map from Fig. $7 \mathrm{a}$ is shown in Fig. $7 \mathrm{~b}$. For a slope measurement of an object surface the procedure is the same. When the shape information of a pyramid is known with phase modulo $2 \pi$ (see Fig. $8 \mathrm{a}$ ), the slope information is obtained by shearing this phase map. Figs. $8 \mathrm{~b}$ and $8 \mathrm{c}$ show the 3D plots of the topography and the slope variation in a direction along one surface of the pyramid, respectively. The slopes of the pyramid are constant on each of the four surfaces. 


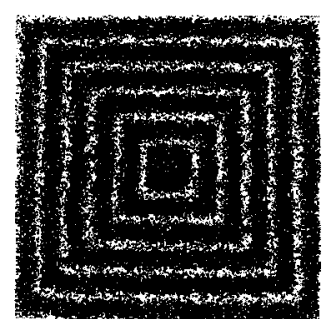

a)

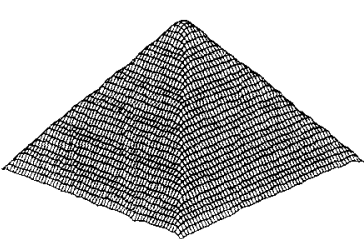

b)

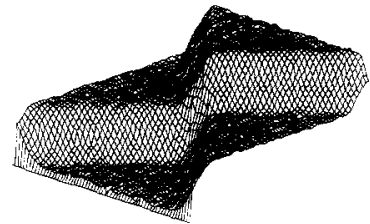

c)

Fig. 8. Slope variation from a shape measurement of a three-dimensional object: (a) phase modulo $2 \pi$; (b) 3D plot of the shape and (c) the $3 \mathrm{D}$ plot of the slope.

\section{Conclusions}

The shearing methods for derivative measurements of a displacement reported in this paper combine several advantages: (i) both the displacement and the derivative information can be obtained from the same data set; (ii) the derivative sensitivity can be controlled after recordings; (iii) displacement derivatives along arbitrary directions can be chosen easily. These techniques can also be applied to a slope measurement of an object surface.

\section{Acknowledgements}

We would like to thank the German Ministry of Research and Development (BMFT) for the financial support and the reviewers for helpful comments.

\section{References}

[1] Y.Y. Hung, Optics Comm. 11 (1974) 132.

[2] Y.Y. Hung, Opt. Eng. 21 (1982) 391.

[3] D.K. Sharma A.R. Ganesan and M.P. Kothiyal, Appl. Optics 27 (1988) 4731.

[4] Y.Y. Hung, in: Speckle Metrology, ed. R.K. Erf (Academic Press, New York, 1978) p. 61.

[5] A. Ettemeyer and M. Honlet, Proc. 1 lth Intern. Congress on Laser in Remote Sensing (Springer, Berlin, 1993) p. 180.

[6] C. Joenathan, R.K. Mohanty and R.S. Sirohi, Appl. Optics 24 (1985) 3106.

[7] P. K. Rastogi, in: Holographic Interferometry (Principles and Methods) ed. P.K. Rastogi (Springer, Berlin, 1994) p. 243.

[8] K. Creath, in: Progress in Optics, XXVI, ed. E. Wolf (Elsevier Science, New York, 1988) p. 366.

[9] U. Schnars and W. Jüptner, Proc. 2nd Intern. Workshop on Automatic Processing of Fringe Patterns (Akademie Verlag, Berlin, 1993) p. 115. 